\title{
EMGNet: Efficient Multi-Scale Feature Generation Adaptive Network
}

\author{
Gwanghan Lee \\ Department of Artificial Intelligence \\ Sungkyunkwan University, South Korea \\ ican0016@g.skku.edu \\ Minha Kim \\ Department of Computer Engineering \\ Sungkyunkwan University, South Korea \\ sunshine01@g.skku.edu
}

\author{
Minha Kim \\ College of Computing and Informatics \\ Sungkyunkwan University, South Korea \\ kimminha@g.skku.edu \\ Simon S. Woo* \\ Department of Applied Data Science \\ Sungkyunkwan University, South Korea \\ swoo@g.skku.edu
}

\begin{abstract}
Recently, an early exit network, which dynamically adjusts the model complexity during inference time, has achieved remarkable performance and neural network efficiency to be used for various applications. So far, many researchers have been focusing on reducing the redundancy of input sample or model architecture. However, they were unsuccessful at resolving the performance drop of early classifiers that make predictions with insufficient high-level feature information. Consequently, the performance degradation of early classifiers had a devastating effect on the entire network performance sharing the backbone. Thus, in this paper, we propose an Efficient Multi-Scale Feature Generation Adaptive Network (EMGNet), which not only reduced the redundancy of the architecture but also generates multi-scale features to improve the performance of the early exit network. Our approach renders multiscale feature generation highly efficient through sharing weights in the center of the convolution kernel. Also, our gating network effectively learns to automatically determine the proper multi-scale feature ratio required for each convolution layer in different locations of the network. We demonstrate that our proposed model outperforms the state-of-the-art adaptive networks on CIFAR10, CIFAR100, and ImageNet datasets. The implementation code is available at https://github.com/lee-gwang/EMGNet
\end{abstract}

\section{CCS CONCEPTS}

- Information systems $\rightarrow$ Data mining; • Computer systems organization $\rightarrow$ Neural networks.

\section{KEYWORDS}

Adaptive Network; Dynamic Neural Networks; Early Exit Networks

*corresponding author

Permission to make digital or hard copies of all or part of this work for personal or classroom use is granted without fee provided that copies are not made or distributed for profit or commercial advantage and that copies bear this notice and the full citation on the first page. Copyrights for components of this work owned by others than ACM must be honored. Abstracting with credit is permitted. To copy otherwise, or republish, to post on servers or to redistribute to lists, requires prior specific permission and/or a fee. Request permissions from permissions@acm.org.

CIKM '21, November 1-5, 2021, Virtual Event, QLD, Australia

(C) 2021 Association for Computing Machinery.

ACM ISBN 978-1-4503-8446-9/21/11 ..\$15.00

https://doi.org/10.1145/3459637.3482337

\section{ACM Reference Format:}

Gwanghan Lee, Minha Kim, Minha Kim, and Simon S. Woo. 2021. EMGNet: Efficient Multi-Scale Feature Generation Adaptive Network. In Proceedings of the 30th ACM International Conference on Information and Knowledge Management (CIKM '21), November 1-5, 2021, Virtual Event, QLD, Australia. ACM, New York, NY, USA, 10 pages. https://doi.org/10.1145/3459637.3482337

\section{INTRODUCTION}

In the past few years, convolutional neural networks (CNN) have demonstrated exceptional performance in various computer vision tasks, such as image recognition [19,28], object detection [23], and semantic segmentation [5, 39]. To further boost the performance, more and more layers are stacked to create deeper architectures. However, such deep and complex architectures lead to a significant increase in parameters and computational cost, naturally complicating model deployment to mobile or Internet-of-Things (IoT) devices. Several researchers have attempted to reduce the computational cost, namely Floating-Point Operations Per Second (FLOPS) and neural network parameters through pruning $[8,10,21,24,25]$, quantization [3, 8, 17], efficient architecture design [7, 12, 15, 42, 43], and knowledge distillation (KD) [1, 11,27].

Different from static approaches, adaptive networks, in which the model complexity can be dynamically adjusted based on the input image [13, 14, 20, 22, 32, 38, 41], have recently gained significant attention. Adaptive networks have successfully reduced the inference time by distinguishing "easy" from "hard" input samples and allocating the appropriate computation power for each sample category. Moreover, among adaptive networks, early exit networks have gained attention, enabling more flexible and efficient prediction of the input samples. Generally, early exit networks are composed of auxiliary classifiers that are attached to different locations of the network. Furthermore, if the prediction confidence for an input sample is higher than an exit criterion in a specific auxiliary classifier, the prediction may end early, completing the given task and thus saving computational cost. However, most previous research on early exit networks have focused only on conditionally reducing either model complexity (e.g., blocks and layers) or input redundancy (e.g., channels and spatial redundancy) for each input image. Also, it has been shown that classifiers located in the early stage of the network tend to yield poor performance due to their lack of high-level features [14, 38]. Eventually, the limitation of shallow classifiers causes an overall performance degradation. 


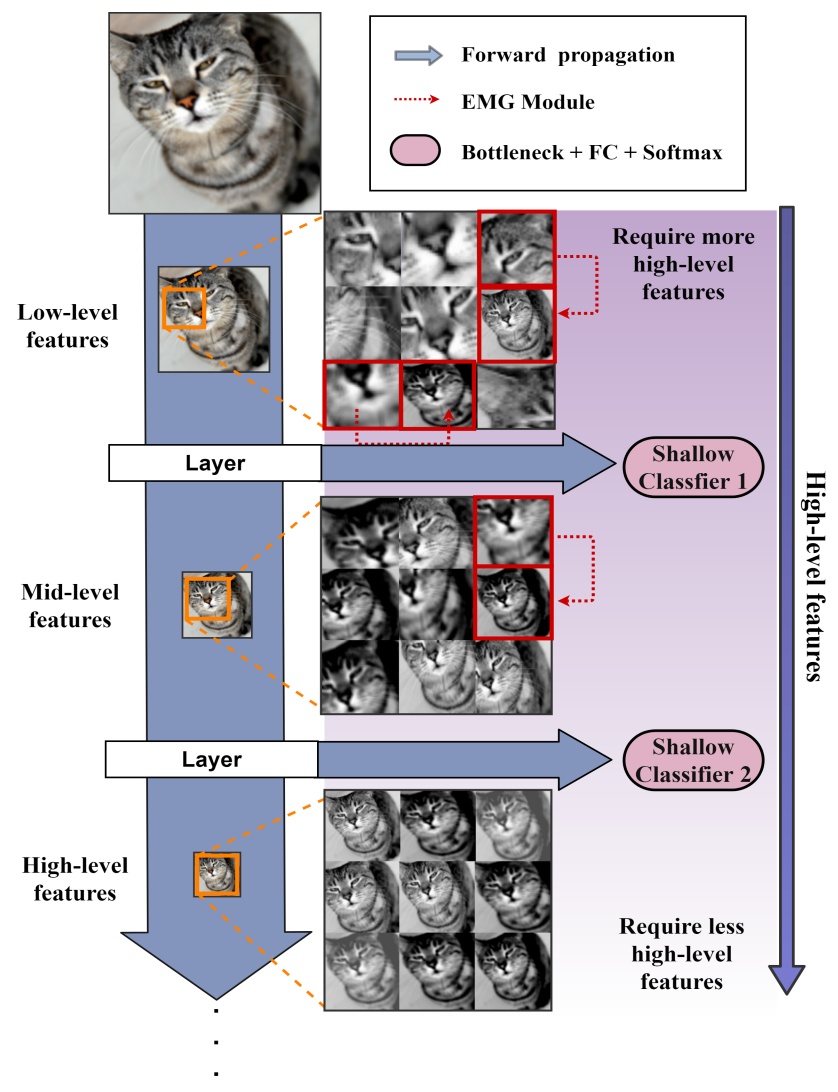

Figure 1: Visualization of the receptive field area considered by various depths of EMGNet. Existing early exit networks lack the information necessary for image prediction because features are used only with fixed-size receptive fields. Ours EMGNet utilizes features of various scales in consideration of multi-size receptive fields through the EMG module.

In this work, we propose a novel approach that not only 'reduces' redundant features but also 'substitutes' them with more meaningful features. The motivation behind our approach stems from the fact that each shallow classifier in the network can exploit multiscale features rather than single-scale features to achieve higher performance. Previously, knowledge distillation (KD) [26, 40] was applied to transfer the knowledge from the final classifier to the shallow classifiers to mitigate the performance degradation of the shallow classifiers. Also, SCANNet [41] added additional attention layers to the auxiliary classifiers. However, only minor or moderate improvements were achieved due to the attention layers that pose an even greater computational cost to the network. Moreover, MSDNet [14] and RANet [38] enhanced the model performance by designing the sub-networks that generate various scale features from multi-resolution representations of the input image. The generated features from each sub-network are further fused to make multi-scale feature maps. However, the ratio of the higher-scale features cannot be dynamically determined and instead is decided in a handcrafted manner [14, 38].

Therefore, in this work, we propose EMGNet, which efficiently generates the multi-scale features to improve the performance of shallow classifiers in the early exit network. The high level concept of our approach is presented in Fig. 1. Similar to other early exit networks [14, 26, 38, 40,41], our EMGNet is composed of a basic CNN backbone network, with several auxiliary classifiers enabling early exits. To effectively create multi-scale features, we only use a single $5 \times 5$ kernel which is specially designed to achieve computational efficiency, without preparing multiple kernel sizes in advance.

In Fig. 2 and 3, we first denote the inner region (9 weights in the center) of the $5 \times 5$ kernel as $3 \times 3$ kernel, then refer to the features extracted using the corresponding $3 \times 3$ kernel as 'Small Receptive Field $(S R F)$ ' features. In contrast, we refer to the features extracted using the fully utilized $5 \times 5$ kernel as 'Large Receptive Field $(L R F)$ ' features, $L R F$ features. Especially, when creating the $L R F$ features, the $S R F$ features extracted in advance can be reused, since it is unnecessary to perform the extra computation for the shared weights of the two kernels. For example, in Fig. 1, due to the large receptive field of the $5 \times 5$ kernel, relatively coarse structures in the input image (e.g., the shape of the cat) are captured. On the other hand, since the receptive field of the $3 \times 3$ kernel is small, structures in finer granularity (e.g., eye or nose of the cat) can be effectively captured.

To summarize, the $5 \times 5$ kernel extracts $L R F$ features, which are coarse features extracted from the deeper layers of the network in general. On the contrary, the $3 \times 3$ kernel extracts $S R F$ features, which are fine-grained features usually extracted from the shallower layers of the network. Hence, in our approach, the multi-scale features consisting of the aforementioned $L R F$ and $S R F$ features are efficiently utilized. Here, we define the term of multi-scale feature ratio as the ratio of the amount of $L R F$ features to the amount of $S R F$ features. In addition, our proposed EMGNet includes a trainable gating network that dynamically determines the adequate multiscale feature ratio for each layer, instead of a handcrafted approach in other research $[14,38]$.

For instance, as shown in Fig. 1, the shallow layers require more high-level features than the deep layers indicating that each layer in the network requires a different multi-scale feature ratio depending on their location in the network. Our proposed novel gating network is capable of dynamically assigning an appropriate multi-scale feature ratio for every individual layer throughout the network. This approach sets us apart from previous approaches such as Inception [29], and MixConv [31] by sharing weights among different kernels. Therefore, our EMGNet does not impose any burden of preparing various sized kernels in advance, significantly improving the reusability and reducing the computational cost.

Our main contributions are summarized into three folds:

- We propose EMGNet, a novel multi-scale feature generation framework in early exit network to enhance the performance of auxiliary classifiers by introducing more meaningful features besides merely reducing the redundant features.

- Our proposed EMGNet efficiently generates multi-scale features by sharing weights between $3 \times 3$ and $5 \times 5$ kernels. Moreover, we introduce a novel trainable gating network to determine the adequate ratio of multi-scale features throughout different layers in the network.

- We compare the performance of our approach with more than ten different similar baselines, including various early 
exit networks. The experimental results show that our method outperforms all the baseline approaches in a resource constrained environment, achieving higher accuracy with less FLOPs.

\section{RELATED WORK}

In this section, we briefly present the related works that are directly relevant to our work on efficient inference deep neural networks along with multi-scale feature maps and networks.

\subsection{Efficient Inference for DNNs}

The need for efficient neural networks that are deployed in a resource constrained environment has been surging, since larger neural nets come with a higher computational cost. So far, major research efforts have been devoted to developing light-weight architectures, such as MobileNet [12], EfficientNet [30], ShuffleNet [42] and GhostNet [7]. On the other hand, efficient network development has been explored by focusing on reducing the network redundancy through pruning $[8,10,21,24,25]$, or quantization $[3,8,17]$. Furthermore, knowledge distillation (KD) [11] was introduced to enhance the performance of a smaller model (student) by transferring the knowledge learned from a larger (teacher) model.

However, different from the prior approaches that act upon input samples statically, adaptive network based approaches [6, 13, 14, $35-38,40,41]$ was proposed. In contrast to the aforementioned approaches, the core idea of an adaptive network is to dynamically adjust the model complexity during inference with respect to the difficulty of test inputs. In particular, some researchers have focused on further reducing the model redundancy in the adaptive network across layers, blocks, or input redundancy in spatial domains or channels. For example, BlockDrop [37] and SkipNet [35] trained the network to skip blocks or layers at run-time per each input sample. Moreover, Hua et al. [13] applied the channel gating block to select only the spatially important region of the input sample to perform convolution operations. In addition, Gao et al. [6] rendered convolution operation fast by dynamically skipping unimportant channels during inference.

Recently, early exiting networks [14, 36, 38, 40, 41] have been proposed, where additional predictors are attached to the various location at the network, enabling some of the input samples to be exited early based on their prediction confidence. To further effectively train the intermediate classifiers, SCAN [41] added an extra attention layer, while Zhang et al. [40] introduced self distillation to transfer knowledge of the final classifier to the intermediate predictors. Moreover, MSDNet [14] and RANet [38] proposed a method of extracting various multi-scale features to create multi-resolution representations of the input images. Then, they fused the extracted features to enhance the performance of intermediate classifiers. In addition, Wang et al. [36] reduced spatial redundancy by training the model to correctly identify the class-discriminative regions from the input images and performing convolution operation only on the class-discriminative parts. However, most of the aforementioned research has solely focused on eliminating redundancy among models or inputs, when designing adaptive networks. On the other hand, in our approach, we not only reduce the redundancy in the adaptive network but also further improve the performance through the creation and use of more effective multi-scale features during inference.

\subsection{Multi-Scale Feature Maps and Multi-Scale Network}

Recent researches have demonstrated notable performance improvements in computer vision tasks using a multi-scale feature map rather than a single-scale feature map. In particular, Inception [29] used a set of different size kernels to extract features of various scales. Also, DenseNet [16] combined the multi-scale features from different layers. As opposed to the vanilla depthwise convolution, MixConv [31] applied different sizes of kernels for depthwise convolution to every other channel. Despite the inconvenience of preparing various sized kernels beforehand, multi-scale features still play a pivotal role in boosting the performance of image recognition tasks. Unfortunately, in case of early exit networks, the lack of high-level features inevitably makes the early classifiers suffer from low performance. Furthermore, poor performance of early classifiers negatively impacts other classifiers, degrading the performance of an entire network. Existing works such as RANet [38] and MSDNet [14] have strived to provide multi-scale features to each layer by extracting features from multi-resolution representations of the input sample. However, these approaches appear to be inefficient because the multi-scale output features are fused together in a handcrafted way.

In our approach, we can use a single size $5 \times 5$ kernel to construct both $3 \times 3$ and $5 \times 5$ kernel through sharing the common weights at the center of $5 \times 5$ kernel. In other words, since the weights are effectively shared in EMGNet, we can adaptively reuse the SRF features to construct LRF features. Also, we proposed the gating network that can be trained to automatically identify the threshold to determine the adequate ratio between the SRF and LRF features at each layer in order to improve the performance of early classifiers in the early exit network.

\section{OUR APPROACH}

In this section, we first briefly explain the main concept of an adaptive inference network. Then, we describe our method of efficiently generating multi-scale features by sharing weights between different size kernels. Lastly, we discuss the overall procedure of training the gating network to determine each layer's adequate multi-scale feature ratio dynamically.

\subsection{Adaptive Inference Network}

We set up an early exit network consisting of $p$ classifiers, and those classifiers are attached to various depths of the network. The network generates $p$ predictions given an input image $x$, which can be represented as follows:

$$
\left[y_{1}, \ldots, y_{p}\right]=f(x ; w)=\left[f_{1}\left(x ; w_{1}\right), \ldots, f_{p}\left(x ; w_{p}\right)\right],
$$

where $f_{p}$ and $w_{p}$ denote the transformation learned by the $p$-th classifier and parameters of the $p$-th classifier, respectively. During inference time, a certain exit criterion is set for each classifier, and when the largest softmax output value of the classifier exceeds the exit criterion, the input image is predicted early. Eventually, the exit criterion can be adjusted to balance the trade-off between 


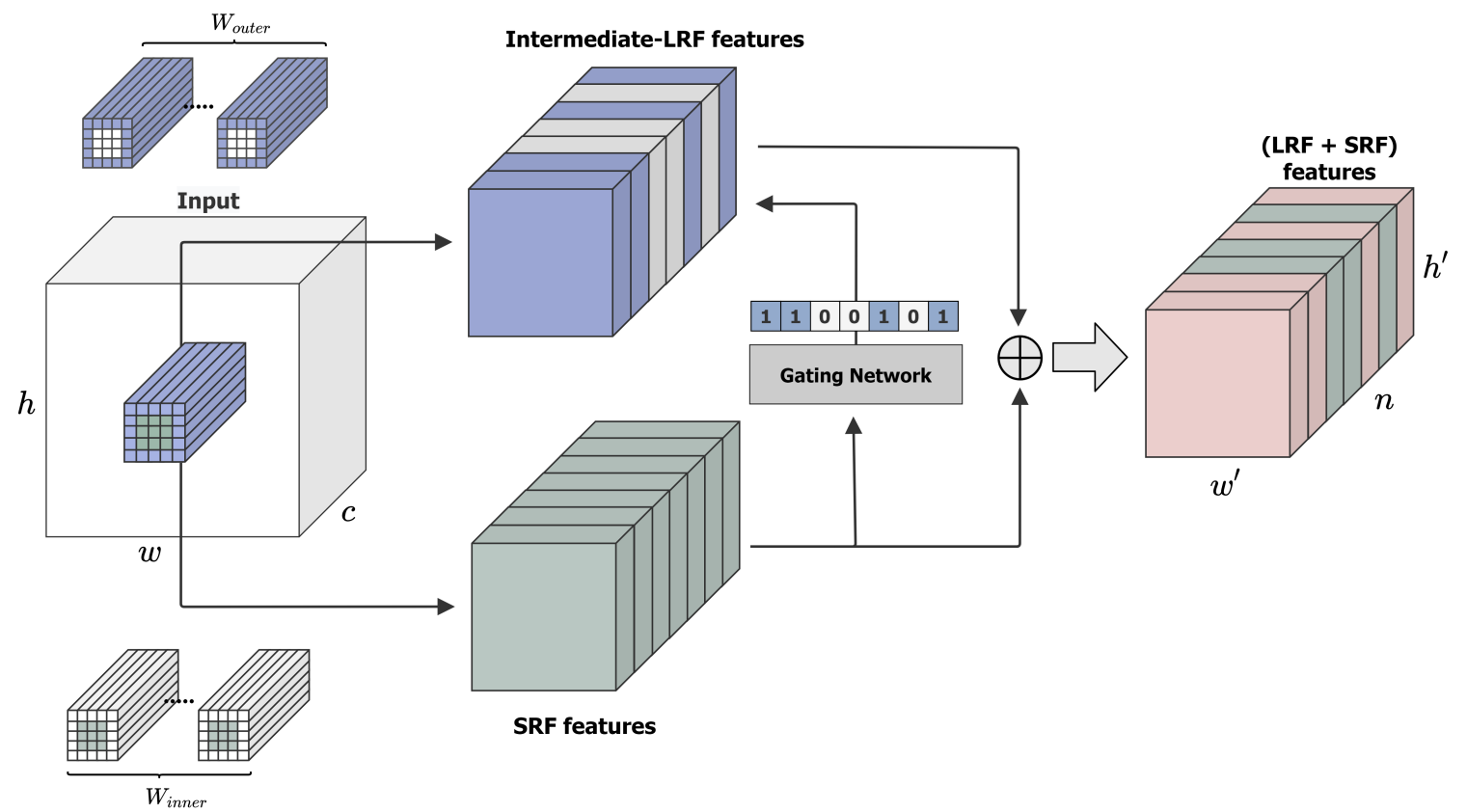

Figure 2: Detailed overview of EMG module. Multi-scale features are generated by performing different convolution operations on each channel using a gating network, where $\oplus$ means the sum of each channel. We effectively generate SRF and LRF features from the single $5 \times 5$ kernel to generate multi-scale (LRF+SRF) features.

classification accuracy and computational cost in inference time, and we can set a series of exit criterion for a computational budget constraint.

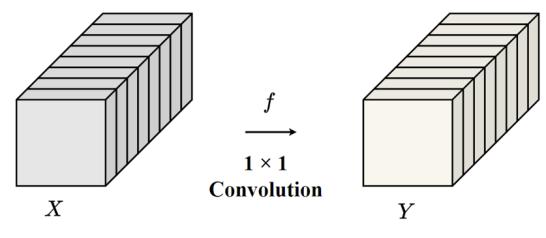

(a) Ordinary convolutional layer.

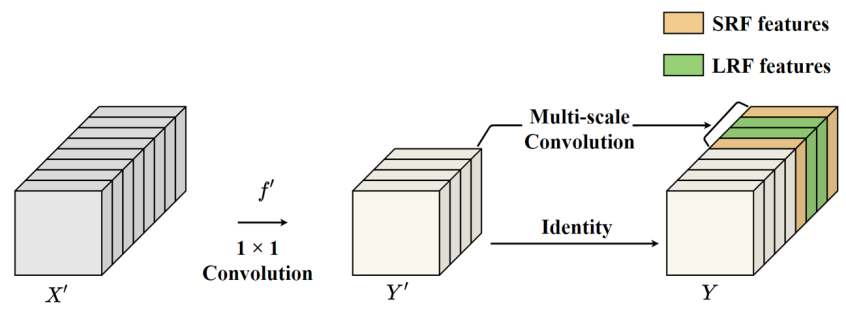

(b) Multi-scale feature generation in EMG module.

Figure 3: Illustrations of (a) Ordinary convolution operation vs. (b) Multi-scale feature generation from our EMG module via two steps.

\subsection{Multi-Scale Feature Generation}

Ordinary convolutions have shown poor performance in early exit networks because they produce highly redundant single-scale feature maps. To overcome this drawback, we propose a novel method of efficiently generating multi-scale features and replacing redundant features with the generated features, consequently improving the performance of early exit classifiers.

Conventional convolution operation. First, let us consider an ordinary convolution layer, which transforms an input feature of width $w$ and height $h$, to an output feature of width $w^{\prime}$ and height $h^{\prime}$. The number of channel of the input and output features are denoted by $c$ and $n$, respectively. Then, the conventional convolution operation can be defined as $Y=X * f$, where $Y \in \mathbb{R}^{n \times w^{\prime} \times h^{\prime}}$, $X \in \mathbb{R}^{c \times w \times h}, f \in \mathbb{R}^{c \times k \times k \times n}$, and $*$, each indicating the output features, input features, convolution filters, and the convolution operation.

EMG module. In contrast to the ordinary $1 \times 1$ convolution, our EMG module includes two steps of convolution operation utilizing various size kernels to extract multi-scale features efficiently. The main difference of our EMG module with conventional convolution is depicted in Fig. 3. In the first step of the EMG module, we create the intermediate feature map by $1 \times 1$ convolution operation using $m$ filters. Unlike the conventional $1 \times 1$ convolution that creates $n$ output channels, our method decreases the number of output channels to $m(m<n)$, thereby reducing the redundancy in the output feature map. We define this initial creation of intermediate feature map as follows: $Y^{\prime}=X * f^{\prime}$, where $Y^{\prime} \in \mathbb{R}^{m \times w^{\prime} \times h^{\prime}}, f^{\prime} \in \mathbb{R}^{c \times 1 \times 1 \times m}$ denotes the output features and convolution filters, respectively. For simplicity, we use $m=0.5 n$ throughout our experiment. Then, 


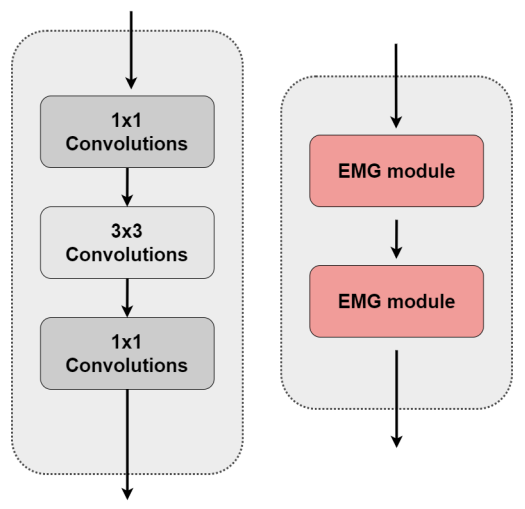

Figure 4: Bottleneck Layer. Left: Ordinary bottleneck layer; Right: Our EMG module replaces $1 \times 1$ convolutional layer. Since we can sufficiently obtain spatial information within the EMG module, we can exclude the $3 \times 3$ convolutional layer.

the second step of generating multi-scale features is defined as follows:

$$
Y_{i}^{\prime \prime}= \begin{cases}\left(Y^{\prime} * W_{\text {inner }}\right)_{i}, & \text { if } D_{i}=0 \\ \left(Y^{\prime} * W_{\text {inner }}+Y^{\prime} * W_{\text {outer }}\right)_{i}, & \text { otherwise }\end{cases}
$$

where $Y^{\prime \prime}$ and $i$ denote the resulting multi-scale output features and the index of output channels, respectively. Herein, $W_{\text {inner }}$ represents the spatial central region of the $5 \times 5$ kernel across the channel dimension, which is reused as $3 \times 3$ kernel as well.

On the other hand, as shown in Fig. $2, W_{\text {outer }}$ denotes the remaining outer part of the $5 \times 5$ kernel, which has not been utilized as $3 \times 3$ kernel. After extracting the SRF features by $W_{\text {inner }}$, the output features are evaluated through a gating network to identify which of the output channels contain meaningful spatial information. Then, the gating network outputs a binary decision tensor $D \in[0,1]^{m}$, assigning either 0 or 1 to each channel. We extract the LRF features only from the output channels assigned 1 by the gating network. The details of the gating network will be explained in the following section.

In our approach, when extracting the LRF, the entire convolution using $5 \times 5 \mathrm{kernel}$ is not required since the center of the $5 \times 5$ kernel is shared with $3 \times 3$ kernel. Only a few extra computations with the outer part of the $5 \times 5 \mathrm{kernel}$ are needed, reducing the computational cost by $9 / 25 \times 100=36 \%$ compared to the conventional $5 \times 5$ convolution. Thus, our approach allows LRF features to be flexibly created by reusing the SRF features extracted from $3 \times 3$ kernel. Finally, as shown in Fig. 3(b), the multi-scale features each extracted from steps 1 and 2 are concatenated to compose the final output features. As shown in Fig. 4, we utilized our proposed EMG module inside a conventional bottleneck architecture by replacing two $1 \times 1$ convolution layers with the EMG modules. Since the substituted EMG module generates a sufficient spatial feature, the $3 \times 3$ convolution layer in the bottleneck architecture is removed.

\subsection{Training EMGNet}

In conventional $\mathrm{CNN}$ models, low-level features tend to be extracted from layers in the early stages of the network, while the high-level features are extracted from layers in proximity to the final classifier. Therefore, in an early exit network, the required ratio for the SRF features varies significantly through different layers in the network to increase the model's overall performance. In our method, the gating threshold is trained to dynamically identify the appropriate proportion of the LRF features constituting the multi-scale feature map for each layer throughout the network.

Trainable a gating network. Our proposed gating network receives the SRF features extracted from $3 \times 3$ kernel, then returns a binary decision tensor $D$, which indicates whether each channel is further used for extracting the LRF features using $5 \times 5$ kernel. Our gating network can be expressed as follows:

$$
\begin{gathered}
H(x)_{i}=\left\{\begin{array}{ll}
1, & \text { if } x_{i} \geq 0 \\
0, & \text { otherwise }
\end{array},\right. \\
S(I, t)_{i}=H\left(\sigma(\text { GlobalAvgPool }(|I|-t)-0.5)_{i}\right), \\
D_{i}=S(I, t)_{i},
\end{gathered}
$$

where $H, S$, and $\sigma$ denotes the binary step function, gate function, and sigmoid function, respectively. Also, the input to the gating network and the threshold tensor are represented by $I=Y^{\prime} * W_{\text {inner }}$ and $t \in \mathbb{R}^{m}$. When predicting the relative importance of each channel in the task of extracting the LRF, the threshold tensor is subtracted from the input features of the gating network. Given the intuition that features composed of higher pixel values are generally more expressive, the channels exceeding the corresponding threshold value on average can be selected as channels to generate the LRF.

In addition, as the binary step function is non-differentiable, we adopt straight-through estimator (STE) [2] to make our gating network compatible with backpropagation.

Final loss function. The aforementioned threshold is able to control the sparsity of the binary decision tensor, thereby consequently determining the ratio between the SRF features and LRF features at each layer. In this work, we introduce a new ratio regularization term, $\mathcal{L}_{r}$ to train the threshold. Also, the penalty $\alpha$ is introduced as a scale factor for the ratio regularization term to regulate the level of the sparsity of the binary decision tensor. To sum up, the final loss function of our network consisting of $p$ classifiers and $E$ layers with EMG module can be represented as follows:

$$
\begin{gathered}
\mathcal{L}_{r}=\sum_{j=1}^{E}\left(\sum_{i=1}^{m} \exp \left(-t_{i j}\right)\right), \\
\mathcal{L}=\sum_{i=1}^{p}\left(\mathcal{L}_{c e}\right)_{i}+\alpha \mathcal{L}_{r},
\end{gathered}
$$

where $\mathcal{L}_{c e}$ is the cross-entropy loss for classification and $\alpha$ is the loss regularization term given as a predetermined hyperparameter. In our experiment, we adjusted $\alpha$ to find the threshold that adequately balances the amount of the SRF and LRF features for any layers. We present the effect of different $\alpha$ values on prediction accuracy in the ablation study. 


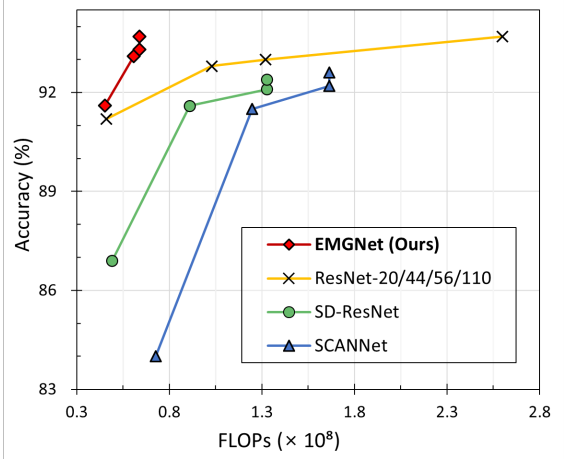

(a) CIFAR-10

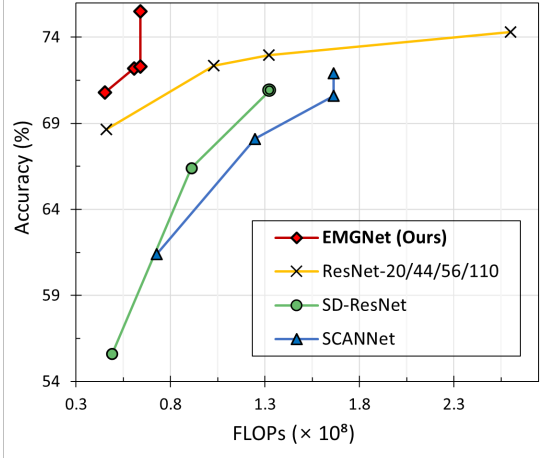

(b) CIFAR-100

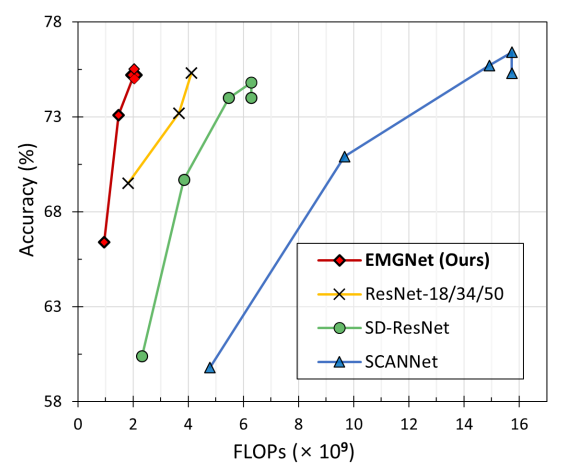

(c) ImageNet

Figure 5: Comparison of accuracy (\%) and floating point operations (FLOPs) of auxiliary classifiers in networks on CIFAR-10, CIFAR-100, and ImageNet, respectively.

\section{EXPERIMENTS AND RESULTS}

In this section, we present our experiments by first describing the datasets we used and then comparing results against baseline approaches. We evaluate our EMGNet on CIFAR-10, CIFAR-100 [18], and ImageNet datasets [4]. To demonstrate the effectiveness of our multi-scale generation method, we use accuracy to measure the detection performance, and floating point operations per second (FLOPs) to estimate the computational cost.

\subsection{Datasets}

CIFAR-10 and CIFAR-100. The CIFAR-10 and CIFAR-100 [18] datasets consist of $32 \times 32$ RGB images each in 10 and 100 classes. The two datasets are both composed of 50,000 images in training set and 10,000 images in testing set. To conduct experiment on adaptive inference network, we hold out 5,000 images from the training set as a validation set to search for the optimal exit criterion for each auxiliary classifier to enable effective early exits.

ImageNet. The ImageNet [4] dataset is a large-scale image dataset consisting of more than $1.2 \mathrm{M} 224 \times 224$ RGB images in 1,000 classes. We also hold out 50,000 images from the training set as a validation set for the adaptive inference experiment.

\subsection{Experimental Settings}

We use the stochastic gradient descent (SGD) optimization with a multi-step learning rate policy. Also, we set the momentum, initial learning rate and the weight decay to $0.9,0.1$, and $1 \times 10^{-4}$, respectively. For the two CIFAR datasets, we train the models using batch size of 64 with 250 epochs and the learning rate divided by a factor of 10 after 125, 188 epochs. For the ImageNet dataset, training is done using batch size of 256 with 90 epochs and the learning rate divided by a factor of 10 after 30,60 epochs. Also, we select $\alpha$ as $1 \times 10^{-5}, 5 \times 10^{-5}, 1 \times 10^{-5}$ throughout different experiments on CIFAR-10, CIFAR-100, and ImageNet. All the experiments are conducted by PyTorch on TITAN RTX GPUs, with 24 GB of dedicated memory. We use Intel Xeon Gold 6230 CPUs with 8 cores each and 256 GB of RAM.

\subsection{Evaluation and Analysis of Auxiliary Classifiers Performance}

To compare performance improvement of each early exit branch of EMGNet, we first evaluate the performance of the individual classifiers and report their accuracy with their corresponding FLOPs, and finally present the final ensemble performance. In our EMGNet, we use ResNet-56 and ResNet-50 as our backbone architecture of the early exit networks. For comparison, we choose the following baseline models to compare the performance of our EMGNet:

- SCANNet [41]. SCANNet is a scalable neural network architecture, which exploits the diversity in prediction difficulty and improves the performance using the self distillation and attention mechanism.

- SD-ResNet [40] . SD-ResNet proposes the self distillation framework to overcome the drawbacks of traditional distillation. The knowledge of the deepest classifier is transferred to shallow classifiers to enhance the performance of the shallow classifiers.

- ResNet [9]. ResNet is the most widely used baseline architecture. We use ResNet with varying depths; ResNet20/44/56/110 for CIFAR and ResNet-18/34/50 for ImageNet, respectively.

We compare the results of each classifier in our EMGNet with the corresponding early exit branches of above baseline models, and report their accuracy with the corresponding FLOPs in Figure 5. In particular, we plot the accuracy vs. FLOPs for the individual classifiers and their final ensemble performance. Our EMGNet is shown in red line, and the baseline SD-ResNet, SCANNet and ResNet are plotted in green, blue, and yellow color, respectively. For CIFAR dataset, the first three points in each line represent the performance of three individual classifier at each early exit branch, where the depth of the classifier increases from left to right. For ImageNet dataset, we have four classifiers, which are shown as first four points, and the last point indicates the final ensemble performance. For ResNet, each point from left to right indicates the performance of ResNet-20, 40, 56, and 110 on CIFAR, and ResNet-18, 34, and 50 for ImageNet, respectively. 
Table 1: Comparison of state-of-the-art efficient inference methods on ImageNet. The checkmark $(\checkmark)$ is used whether each approach utilizes the early exit, dynamic inference, or pruning. The best performance are highlighted in bold.

\begin{tabular}{l|ccc|c|c}
\hline \multirow{2}{*}{\multicolumn{1}{c|}{ Model }} & \multicolumn{3}{|c|}{ Method } & \multirow{2}{*}{ FLOPs $\left(\times 10^{9}\right)$} & \multirow{2}{*}{ Top-1 Acc. (\%) } \\
\cline { 2 - 4 } & Early Exit & Dynamic & Pruning & & 74.9 \\
\hline Resnet50 0.75× [9] & $\times$ & $\times$ & $\times$ & 2.3 & 75.0 \\
GhostNet [7] & $\times$ & $\times$ & $\times$ & 2.2 & 75.3 \\
\hline BlockDrop [37] & $\times$ & $\checkmark$ & $\times$ & 9.9 & 74.9 \\
URNet [20] & $\times$ & $\checkmark$ & $\times$ & 9.8 & 74.2 \\
SkipNet [35] & $\times$ & $\checkmark$ & $\times$ & 3.0 & 73.3 \\
\hline CP [10] & $\times$ & $\times$ & $\checkmark$ & 2.0 & 74.7 \\
ThiNet-50 [25] & $\times$ & $\times$ & $\checkmark$ & 2.1 & 75.6 \\
\hline DynConv [34] & $\times$ & $\checkmark$ & $\checkmark$ & 3.0 & 75.5 \\
ConvNet-AIG-50 [33] & $\times$ & $\checkmark$ & $\checkmark$ & 2.6 & 74.3 \\
\hline SD-ResNet [40] & $\checkmark$ & $\checkmark$ & $\times$ & 4.2 & 75.1 \\
RANet [38] & $\checkmark$ & $\checkmark$ & $\times$ & 2.0 & $\mathbf{7 5 . 6}$ \\
EMGNet (Ours) & $\checkmark$ & $\checkmark$ & $\times$ & $\mathbf{2 . 1}$ & \\
\hline
\end{tabular}

Results on CIFAR-10. First, results from CIFAR-10 dataset is presented in Figure. 5(a). We can observe that EMGNet outperforms the accuracy of SD-ResNet by $2.2 \%$ on average for each classifier, ranging from $1.2 \%$ to $4.7 \%$, with the same FLOPs. Also, the average accuracy gain of $2.9 \%$ is achieved over SCANNet, ranging from $1.1 \%$ to $7.6 \%$. Furthermore, on the ensemble of all classifiers, EMGNet shows higher accuracies than SD-ResNet and SCANNet by $1.1 \%$ and $1.3 \%$ respectively, while maintaining lower FLOPs than other methods. In addition, our approach clearly outperforms ResNet-20/44/56/100 at every exit branch. The results demonstrate that our early exit classifiers better identify more meaningful features and correctly predict more images early, resulting in higher efficiency with more accurate predictions.

Results on CIFAR-100. Figure. 5(b) presents the experimental result on CIFAR-100 dataset. Similarly, our approach performs better than other baseline models. Comparing our individual classifiers with those in SD-ResNet, we achieve $6.8 \%$ accuracy gain on average, varying from $1.4 \%$ to $15.2 \%$. EMGNet is superior to SCANNet as well, where our EMGNet achieves $4.7 \%$ higher accuracy on average for each classifier, ranging from $1.7 \%$ to $9.4 \%$. Furthermore, EMGNet reduces FLOPs for all classifiers by $0.04 \times 10^{8}-0.69 \times 10^{8}$ compared to SD-ResNet, and by $0.27 \times 10^{8}-1.02 \times 10^{8}$ compared to SCANNet. To summarize, on average, our EMGNet shows accuracy gain of $6.8 \%$ and $4.7 \%$, while significantly reducing FLOPs by $44.51 \%$ and $58.09 \%$ than SD-ResNet and SCANNet, respectively.

Results on ImageNet. As a baseline model, we use ResNet18/34/50 [9], each of which being a comparable early exit counterpart with our EMGNet backbone. As shown in Figure. 5(c), our EMGNet exceeds the accuracy of individual classifiers of SDResNet by $2.46 \%$ on average, ranging from $0.2 \%$ to $6 \%$. Moreover, EMGNet also outperforms SCANNet with an average accuracy gain of $1.42 \%$, from $0.2 \%$ to $6.6 \%$. Our EMGNet also achieves the lowest FLOPs on average, substantially reducing FLOPs by $44.91 \%$ and 73.22\% than SD-ResNet and SCANNet, respectively.

Although the fourth classifier of SCANNet shows slightly higher accuracy than our EMGNet, our approach significantly cut down on FLOPs by around $87 \%$ of SCANNet. This indicates that our EMGNet strikes a better balance between computational cost and accuracy in each classifier, compared to the SCANNet. In other words, owing to our EMG module, EMGNet can achieve significantly low FLOPs compared to other state-of-the-art baseline networks.

Analysis on Improved accuracy and Reduced FLOPs. We observe that our EMGNet shows significantly lower FLOPs than SD-ResNet and SCANNet for the same prediction accuracy. Applying additional attention mechanisms leads to unnecessary computations, resulting in high FLOPs in SCANNet. Moreover, selfdistillation hardly improve the performance of early classifiers in SD-ResNet. We believe that to effectively reduce the computational cost while maintaining the accuracy, the early classifiers should predict more input images with higher accuracy, thereby exiting many images early. Our EMGNet is clearly designed to provide such effects and achieve the lowest FLOPs on average by enhancing the prediction accuracy of early classifiers. Moreover, we demonstrate that EMGNet achieves superior accuracy on the ensemble of all classifiers without any accuracy drop. This demonstrates the effectiveness of our weight sharing method, which successfully reduces the computational cost involved in separate convolution operations using different size kernels.

\subsection{Evaluation and Analysis of Adaptive Inference Performance}

In this section, we present the experimental results to demonstrate the effectiveness of our EMGNet for adaptive inference performance in a resource constrained environment. We use ImageNet dataset for evaluation, and ten different baseline methods, including various efficient inference techniques, as follows:

- ResNet50 0.75 $\times$ [9]. The number of input and output channels in the ordinary ResNet50 is reduced using the width multiplier $\alpha=0.75$.

- GhostNet [7]. GhostNet is proposed to reduce redundancy among feature maps. They utilized cheap linear operations on a portion of the ordinary output channels to generate new intrinsic features.

- BlockDrop [37]. The goal of BlockDrop is to dynamically skip layers by applying the reinforcement learning mechanism. 


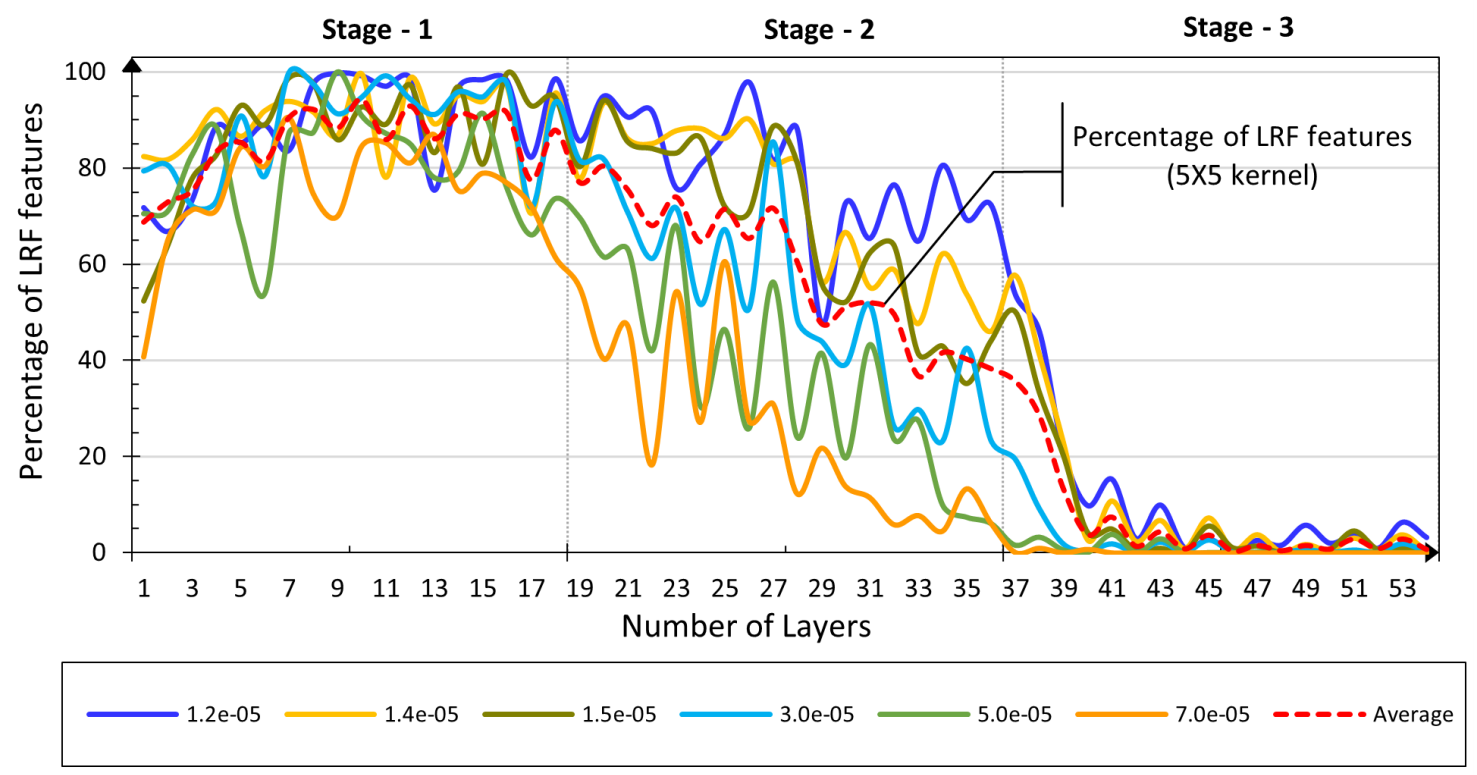

Figure 6: We present the percentage of LRF features in each layer of EMGNet with CIFAR-10. The $5 \times 5$ kernels are shared with the $3 \times 3$ kernels to allow reuse and reduce complexity, and we vary six different $\alpha$ values, and present the average of the percentage of LRF features.

- URNet [20]. URNet is a resizable residual network to dynamically adjust the model complexity as required during inference.

- SkipNet [35]. SkipNet utilizes both supervised and reinforcement learning to make skipping decisions on each layer in the network.

- CPNet [10]. CPNet prunes channels at runtime, rendering the convolution operation fast by reducing channel redundancy.

- ThiNet-50 [25]. ThiNet is proposed to reduce the filter redundancy. After sorting the filters based on the relative importance score, the filters ranked low are removed.

- DynConv [34]. DynConv dynamically decides the spatially essential regions in the input image to conduct convolution operation on.

- ConvNet-AIG-50 [33]. ConvNet-AIG adaptively decides which layers to execute by leveraging a small gate for each layer.

- RANet [38]. RANet utilizes multi-resolution representations of input images to generate multi-scale features that enable effective early exit.

In particular, we used the budgeted batch classification setting [14, 38] and set computational budgets by adjusting the exit criterion.

Comparison with baseline methods. In overall, our EMGNet achieves $0.7 \%$ and $0.6 \%$ higher accuracy with having $8.7 \%$, and $4.46 \%$ less FLOPs than ResNet [9] and GhostNet [7], respectively. Moreover, when comparing with BlockDrop [37], URNet [20], and SkipNet [35], EMGNet achieves the highest accuracy of 75.6\% each using $21.4 \%, 21.2 \%$, and $70 \%$ less FLOPs than those of the baselines. In particular, EMGNet also significantly outperforms the static pruning networks such as CP [10] and ThiNet [25], showing $2.3 \%$ and $0.9 \%$ higher accuracy under similar computational cost constraints. Likewise, EMGNet outperforms the dynamic pruning networks such as DynConv [34] and ConvNet-AIG-50 [33], reducing FLOPs by $30 \%$ and $20 \%$ without any loss in accuracy. Our final experiment with SD-ResNet [40] and RANet [38], which are the two state-of-the-art baseline early exit networks, demonstrates that EMGNet exceeds the accuracy of the baselines by $0.5 \%-1.3 \%$ under either similar or less strict computation constraints, demonstrating our approach is computationally more effective, while maintaining the highest performance.

\section{DISCUSSION}

In this section, we first discuss the effect of $\alpha$ on accuracy. Next, we decompose and visualize the multi-scale feature patterns at each layer across different positions in the network. Finally, we empirically demonstrate the strength of our approach through ablation study, by comparing the accuracy of each classifier with and without multi-scale features that we proposed.

\subsection{Hyperparameter Search for $\alpha$}

As explained in Section 3.3, $\alpha$ is a hyperparameter that controls the magnitude of the threshold in order to regulate multi-scale feature ratio across the network. To find the proper threshold during training, we experiment with various values from $5 \times 10^{-6}$ to $5 \times 10^{-5}$ for $\alpha$. We then select $\alpha$ based on the accuracy of the last classifier, and finally chose $1 \times 10^{-5}$ as $\alpha$ for CIFAR-10 dataset as shown in Table 2. Similarly, hyperparameter $\alpha$ for CIFAR-100 and ImageNet datasets is chosen as $5 \times 10^{-5}$ and $1 \times 10^{-5}$, respectively. 
Table 2: Top-1 Accuracy (\%) results with various $\alpha$. In order to regulate multi-scale feature ratio across the EMGNet, we experiment various values of $\alpha$, with CIFAR10.

\begin{tabular}{c|ccccccccc}
\hline $\boldsymbol{\alpha}$ & $\mathbf{5 . 0 e - 0 6}$ & $\mathbf{5 . 2 e - 0 6}$ & $\mathbf{5 . 4 e - 0 6}$ & $\mathbf{5 . 8 e - 0 6}$ & $\mathbf{1 . 0 e - 0 5}$ & $\mathbf{1 . 2 e - 0 5}$ & $\mathbf{1 . 8 e - 0 5}$ & $\mathbf{3 . 0 e - 0 5}$ & $\mathbf{5 . 0 e - 0 5}$ \\
\hline \hline Top-1 Acc. (\%) & 93.22 & 92.73 & 92.61 & 93.26 & $\mathbf{9 3 . 3 3}$ & 93.04 & 92.85 & 92.8 & 92.66 \\
\hline
\end{tabular}

Table 3: Top-1 Accuracy (\%) using the single-scale features from fixed size $3 \times 3$ (M1) and $5 \times 5$ kernel (M2) as well as multi-scale features using uniform ratio on CIFAR-10 dataset (M3), where our approach (M4) dynamically adjusts the use of different kernels and generates more efficient multi-scale features.

\begin{tabular}{|c|c|c|c|c|}
\hline Top-1 Accuracy (\%) & $\begin{array}{c}\text { Early exit } \\
\text { Classifier } 1\end{array}$ & $\begin{array}{c}\text { Early exit } \\
\text { Classifier } 2\end{array}$ & $\begin{array}{c}\text { Early exit } \\
\text { Classifier } 3\end{array}$ & $\begin{array}{c}\text { Final } \\
\text { Ensemble }\end{array}$ \\
\hline M1. Fixed 3×3 kernel (Single-scale features) & 89.30 & 92.65 & 92.96 & 93.46 \\
\hline M2. Fixed $5 \times 5$ kernel (Single-scale features) & 87.64 & 92.52 & 91.33 & 92.49 \\
\hline M3. Uniform Multi-scale features (the ratio between $3 \times 3$ and $5 \times 5$ kernel $=50: 50$ ) & 89.33 & 91.43 & 92.02 & 92.57 \\
\hline M4. Dynamic Multi-scale features (Ours) & 91.57 & 93.05 & 93.33 & 93.70 \\
\hline
\end{tabular}

\subsection{Percentage of Generated Large Receptive Field (LRF) features at each layer}

We analyze how our proposed LRF features are generated and used at each layer. Figure. 6 presents the percentage of the LRF features produced by our EMG module throughout the network in the Y-axis. And the X-axis represent each layer in the network. We apply six different $\alpha$ values to evaluate on CIFAR-10 dataset.

We can observe that the amount of generated the LRF features tend to decrease, as the depth of the layer increases. This in turn can be interpreted as the lack of coarse feature information resulting in generating more LRF, especially for the shallow (early) layers in Stage-1 and 2. On the other hand, deeper layers with sufficient coarse feature information do not require additional generation of extra LRF features using $5 \times 5$ kernel. Also, the magnitude of these patterns is scaled by $\alpha$, but the scaling does not affect the overall trend significantly. Therefore, it seems that our gating network adequately supplies multi-scale features per layer.

\subsection{Ablation study on the Effect of Multi-Scale Features.}

To demonstrate the effect of introducing multi-scale features at each layer, we conduct an ablation study to measure the Top-1 accuracy at each early exit classifier on CIFAR-10 dataset. As shown in Table 3, we first generate the single-scale features using the $3 \times 3$ (M1) and $5 \times 5$ kernel (M2), as shown in the 2nd and 3rd row. These can characterize how fixed single-scale features perform against our proposed multi-scale features.

In addition, we experiment the uniform multi-scale feature generation (M3), where the ratio between $3 \times 3$ and $5 \times 5$ kernel $=50: 50$. Hence, M3 captures the constant use of the SRF and LRF features without dynamically adjusting it, throughout the network. In contrast, our approach (M4) dynamically control the use of $3 \times 3$ and $5 \times 5$ kernel through trainable gating network at each layer, as depicted in Figure 6. As shown in Table 3, our approach M4 achieves the highest accuracy at every early exit classifier. Also, the final ensemble result also confirms that ours is the highest. Therefore, this ablation study clearly demonstrates that our dynamic use of SRF and LRF features when creating multi-scale features, is in fact more effective over single-scale features or uniform multi-scale features.

\section{CONCLUSION}

Prior early exit networks focus only on reducing redundancy of models or inputs, resulting in poor performance at the early classifiers. The low performance of early classifiers prevents early prediction of more image samples and thus increases computational cost. In this work, we propose our novel EMGNet to improve the performance of early classifiers that lack SRF information in a highly efficient manner. Adequately generated multi-scale features at each layer with our proposed EMG module improve the entire network performance. Therefore, we show that multi-scale feature generation mechanism not only enables early prediction of more input samples compared to existing early exit networks, but also shows higher performance in a resource-constrained environments compared to other state-of-the-art adaptive network baselines.

For future work, we plan to experiment additional datasets and evaluate our approach for various ML tasks. Also, we plan to actually deploy our EMGNet on hardware and measure actual hardware latency performance in addition to FLOPs. Finally, we plan to release our source code openly after acceptance of our paper.

\section{ACKNOWLEDGMENTS}

This work was partly supported by Institute of Information \& communications Technology Planning \& Evaluation (IITP) grant funded by the Korea government (MSIT) (No.2019-0-00421, AI Graduate School Support Program (Sungkyunkwan University)), (No. 2019-001343, Regional strategic industry convergence security core talent training business) and the Basic Science Research Program through National Research Foundation of Korea (NRF) grant funded by Korea government MSIT (No. 2020R1C1C1006004). Also, this research was partly supported by IITP grant funded by the Korea government MSIT (No. 2021-0-00017, Original Technology Development of Artificial Intelligence Industry) and was partly supported by the Korea government MSIT, under the High-Potential Individuals Global Training Program (2020-0-01550) supervised by the IITP. 


\section{REFERENCES}

[1] Lei Jimmy Ba and Rich Caruana. 2013. Do deep nets really need to be deep? arXiv preprint arXiv:1312.6184 (2013).

[2] Yoshua Bengio, Nicholas Léonard, and Aaron Courville. 2013. Estimating or propagating gradients through stochastic neurons for conditional computation. arXiv preprint arXiv:1308.3432 (2013).

[3] Wenlin Chen, James Wilson, Stephen Tyree, Kilian Q Weinberger, and Yixin Chen. 2016. Compressing convolutional neural networks in the frequency domain. In Proceedings of the 22nd ACM SIGKDD International Conference on Knowledge Discovery and Data Mining. 1475-1484.

[4] Jia Deng, Wei Dong, Richard Socher, Li-Jia Li, Kai Li, and Li Fei-Fei. 2009. Imagenet: A large-scale hierarchical image database. In 2009 IEEE conference on computer vision and pattern recognition. Ieee, 248-255.

[5] Qi Dou, Hao Chen, Yueming Jin, Lequan Yu, Jing Qin, and Pheng-Ann Heng. 2016. 3D deeply supervised network for automatic liver segmentation from CT volumes. In International conference on medical image computing and computer-assisted intervention. Springer, 149-157.

[6] Xitong Gao, Yiren Zhao, Łukasz Dudziak, Robert Mullins, and Cheng-zhong $\mathrm{Xu}$. 2018. Dynamic channel pruning: Feature boosting and suppression. arXiv preprint arXiv:1810.05331 (2018).

[7] Kai Han, Yunhe Wang, Qi Tian, Jianyuan Guo, Chunjing Xu, and Chang Xu. 2020 Ghostnet: More features from cheap operations. In Proceedings of the IEEE/CVF Conference on Computer Vision and Pattern Recognition. 1580-1589.

[8] Song Han, Huizi Mao, and William J Dally. 2015. Deep compression: Compressing deep neural networks with pruning, trained quantization and huffman coding. arXiv preprint arXiv:1510.00149 (2015).

[9] Kaiming He, Xiangyu Zhang, Shaoqing Ren, and Jian Sun. 2016. Deep residual learning for image recognition. In Proceedings of the IEEE conference on computer vision and pattern recognition. $770-778$.

[10] Yihui He, Xiangyu Zhang, and Jian Sun. 2017. Channel pruning for accelerating very deep neural networks. In Proceedings of the IEEE International Conference on Computer Vision. 1389-1397.

[11] Geoffrey Hinton, Oriol Vinyals, and Jeff Dean. 2015. Distilling the knowledge in a neural network. arXiv preprint arXiv:1503.02531 (2015).

[12] Andrew G Howard, Menglong Zhu, Bo Chen, Dmitry Kalenichenko, Weijun Wang, Tobias Weyand, Marco Andreetto, and Hartwig Adam. 2017. Mobilenets Efficient convolutional neural networks for mobile vision applications. arXiv preprint arXiv:1704.04861 (2017).

[13] Weizhe Hua, Yuan Zhou, Christopher De Sa, Zhiru Zhang, and G Edward Suh. 2018. Channel gating neural networks. arXiv preprint arXiv:1805.12549 (2018).

[14] Gao Huang, Danlu Chen, Tianhong Li, Felix Wu, Laurens van der Maaten, and Kilian Q Weinberger. 2017. Multi-scale dense networks for resource efficient image classification. arXiv preprint arXiv:1703.09844 (2017).

[15] Gao Huang, Shichen Liu, Laurens Van der Maaten, and Kilian Q Weinberger. 2018. Condensenet: An efficient densenet using learned group convolutions. In Proceedings of the IEEE conference on computer vision and pattern recognition. $2752-2761$.

[16] Gao Huang, Zhuang Liu, Laurens Van Der Maaten, and Kilian Q Weinberger. 2017. Densely connected convolutional networks. In Proceedings of the IEEE conference on computer vision and pattern recognition. 4700-4708.

[17] Itay Hubara, Matthieu Courbariaux, Daniel Soudry, Ran El-Yaniv, and Yoshua Bengio. 2016. Binarized neural networks. In Proceedings of the 30th International Conference on Neural Information Processing Systems. 4114-4122.

[18] Alex Krizhevsky, Geoffrey Hinton, et al. 2009. Learning multiple layers of features from tiny images. (2009).

[19] Alex Krizhevsky, Ilya Sutskever, and Geoffrey E Hinton. 2012. Imagenet classification with deep convolutional neural networks. Advances in neural information processing systems 25 (2012), 1097-1105.

[20] Sangho Lee, Simyung Chang, and Nojun Kwak. 2020. URNet: User-Resizable Residual Networks with Conditional Gating Module. In Proceedings of the AAAI Conference on Artificial Intelligence, Vol. 34. 4569-4576.

[21] Hao Li, Asim Kadav, Igor Durdanovic, Hanan Samet, and Hans Peter Graf. 2016 Pruning filters for efficient convnets. arXiv preprint arXiv:1608.08710 (2016).

[22] Ji Lin, Yongming Rao, Jiwen Lu, and Jie Zhou. 2017. Runtime neural pruning. In Proceedings of the 31st International Conference on Neural Information Processing Systems. $2178-2188$.
[23] Wei Liu, Dragomir Anguelov, Dumitru Erhan, Christian Szegedy, Scott Reed, Cheng-Yang Fu, and Alexander C Berg. 2016. Ssd: Single shot multibox detector. In European conference on computer vision. Springer, 21-37.

[24] Zhuang Liu, Jianguo Li, Zhiqiang Shen, Gao Huang, Shoumeng Yan, and Changshui Zhang. 2017. Learning efficient convolutional networks through network slimming. In Proceedings of the IEEE International Conference on Computer Vision. 2736-2744.

[25] Jian-Hao Luo, Jianxin Wu, and Weiyao Lin. 2017. Thinet: A filter level pruning method for deep neural network compression. In Proceedings of the IEEE international conference on computer vision. 5058-5066.

[26] Mary Phuong and Christoph H. Lampert. 2019. Distillation-Based Training for Multi-Exit Architectures. In Proceedings of the IEEE/CVF International Conference on Computer Vision (ICCV).

[27] Adriana Romero, Nicolas Ballas, Samira Ebrahimi Kahou, Antoine Chassang, Carlo Gatta, and Yoshua Bengio. 2014. Fitnets: Hints for thin deep nets. arXiv preprint arXiv:1412.6550 (2014).

[28] Karen Simonyan and Andrew Zisserman. 2014. Very deep convolutional networks for large-scale image recognition. arXiv preprint arXiv:1409.1556 (2014).

[29] Christian Szegedy, Vincent Vanhoucke, Sergey Ioffe, Jon Shlens, and Zbigniew Wojna. 2016. Rethinking the inception architecture for computer vision. In Proceedings of the IEEE conference on computer vision and pattern recognition. 2818-2826.

[30] Mingxing Tan and Quoc Le. 2019. Efficientnet: Rethinking model scaling for convolutional neural networks. In International Conference on Machine Learning. PMLR, 6105-6114.

[31] Mingxing Tan and Quoc V Le. 2019. Mixconv: Mixed depthwise convolutional kernels. arXiv preprint arXiv:1907.09595 (2019).

[32] Andreas Veit and Serge Belongie. 2018. Convolutional networks with adaptive inference graphs. In Proceedings of the European Conference on Computer Vision (ECCV). 3-18.

[33] Andreas Veit and Serge Belongie. 2018. Convolutional networks with adaptive inference graphs. In Proceedings of the European Conference on Computer Vision (ECCV). 3-18.

[34] Thomas Verelst and Tinne Tuytelaars. 2020. Dynamic convolutions: Exploiting spatial sparsity for faster inference. In Proceedings of the IEEE/CVF Conference on Computer Vision and Pattern Recognition. 2320-2329.

[35] Xin Wang, Fisher Yu, Zi-Yi Dou, Trevor Darrell, and Joseph E Gonzalez. 2018. Skipnet: Learning dynamic routing in convolutional networks. In Proceedings of the European Conference on Computer Vision (ECCV). 409-424.

[36] Yulin Wang, Kangchen Lv, Rui Huang, Shiji Song, Le Yang, and Gao Huang. 2020. Glance and focus: a dynamic approach to reducing spatial redundancy in image classification. arXiv preprint arXiv:2010.05300 (2020).

[37] Zuxuan Wu, Tushar Nagarajan, Abhishek Kumar, Steven Rennie, Larry S Davis, Kristen Grauman, and Rogerio Feris. 2018. Blockdrop: Dynamic inference paths in residual networks. In Proceedings of the IEEE Conference on Computer Vision and Pattern Recognition. 8817-8826.

[38] Le Yang, Yizeng Han, Xi Chen, Shiji Song, Jifeng Dai, and Gao Huang. 2020. Resolution adaptive networks for efficient inference. In Proceedings of the IEEE/CVF Conference on Computer Vision and Pattern Recognition. 2369-2378.

[39] Lequan Yu, Xin Yang, Hao Chen, Jing Qin, and Pheng Ann Heng. 2017. Volumetric ConvNets with mixed residual connections for automated prostate segmentation from 3D MR images. In Proceedings of the AAAI Conference on Artificial Intelligence, Vol. 31.

[40] Linfeng Zhang, Jiebo Song, Anni Gao, Jingwei Chen, Chenglong Bao, and Kaisheng Ma. 2019. Be your own teacher: Improve the performance of convolutional neural networks via self distillation. In Proceedings of the IEEE/CVF International Conference on Computer Vision. 3713-3722.

[41] Linfeng Zhang, Zhanhong Tan, Jiebo Song, Jingwei Chen, Chenglong Bao, and Kaisheng Ma. 2019. SCAN: A scalable neural networks framework towards compact and efficient models. arXiv preprint arXiv:1906.03951 (2019).

[42] Xiangyu Zhang, Xinyu Zhou, Mengxiao Lin, and Jian Sun. 2018. Shufflenet: An extremely efficient convolutional neural network for mobile devices. In Proceedings of the IEEE conference on computer vision and pattern recognition. 6848-6856.

[43] Barret Zoph, Vijay Vasudevan, Jonathon Shlens, and Quoc V Le. 2018. Learning transferable architectures for scalable image recognition. In Proceedings of the IEEE conference on computer vision and pattern recognition. 8697-8710. 